\title{
Summary: current theories and directions of strangeness signals for quark matter
}

\author{
H Stöcker \\ Institut für Theoretische Physik, Postfach 1119 32, D-60054 Frankfurt am Main, Germany
}

Received 26 September 1997

Abstract. A summary of the theory part of the Strangeness and Quark Matter Symposium is given.

\section{Preface}

The marvellous island of Santorini with its violent volcano eruption in $1500 \mathrm{BC}$ is uniquely fit to bring theorists and experimentalists together to exchange thoughts about phase transitions and the properties of strange matter.

The phase transitions in historic times have had a tremendous impact on the natural and cultural life in the Aegean. Now, the archaeologists also try to reconstruct the dynamics which has taken place in that non-equilibrium process through analysing the existing observables a long time after the interesting event.

However, there is an important difference between the situations of the archaeologists and physicists: whereas there is no doubt that a volcano eruption has taken place, it is not clear whether a phase transition to the quark-gluon phase happens in a heavy ion reaction or not.

In the following, I summarize the theoretical contributions to our meeting, starting from the talks dealing with hadron abundances. Most of the talks assume thermodynamic and (partial) chemical equilibrium. With these premises, experimental data can be fitted surprisingly well. Medium effects may play an important role in hot and dense matter, as well as in the interior of neutron stars. This is discussed in section 2.1. Since it is unclear whether thermodynamical equilibrium is achieved in a heavy-ion reaction, non-equilibrium dynamical models have been developed. Contributions based on these are summarized in section 2.2. The astrophysical applications will be presented in section 3. Research on the exciting possibility to produce exotic forms of strange hadronic or quark matter and their detectability in heavy-ion experiments are discussed in sections 4 and 5.

\section{Strange hadron abundances}

An impressive talk was given by Becattini in which he showed that hadron abundances in $\mathrm{e}^{+} \mathrm{e}^{-}, \mathrm{pp}$ and $\mathrm{p} \overline{\mathrm{p}}$ collisions are very well described if it is assumed that particles originate in hadron gas fireballs at thermal and partial chemical equilibrium. Besides the two parameters which are used in a canonical formalism of statistical mechanics, an additional parameter $\gamma_{\mathrm{s}}$ is used which accounts for incomplete saturation of strange particles. The fit value of less then one indicates an incomplete chemical equilibrium of strangeness. The success of 
the fit indicates that hadron production in elementary high-energy collisions is dominated by phase-space rather than by microscopic dynamics.

The extrapolation of this finding to heavy-ion collisions, however, is not valid: a simple dynamical hadronization scheme, where thermodynamic equilibrium between a quark blob and the hadron layer is imposed, reveals a more complex picture. Particle ratios can be reproduced nicely with the same number of parameters as in the static ansatz of a hadron gas in equilibrium, while the spacetime evolution of the system shows strong changes of the strange and baryo-chemical potentials due to baryon and strangeness distillery [1,2]. It seems questionable whether final particle yields reflect the actual thermodynamic properties of the system at a certain stage of the evolution.

So far all models have failed to describe the recently reported unusually high $\bar{\Lambda} / \overline{\mathrm{p}}$ ratio of approximately 3 to 5 . One possible explanation would be that $\bar{\Lambda}$ have a far lower annihilation cross section than $\overline{\mathrm{p}}$. Assuming that $\overline{\mathrm{p}}$ and $\bar{\Lambda}$ are both produced according to a thermal model, this difference in the annihilation cross section would account for the dramatic $\bar{\Lambda}$ enhancement. A straightforward way to test this ansatz would be the measurement of $\bar{p}$ and $\bar{\Lambda}$ flow. For $\bar{p}$ flow a strong anti-correlation with regard to the 'conventional' baryon flow is observed due to their large annihilation cross section. The same would only hold true for $\bar{\Lambda}$ if their annihilation cross section is similarly large. If, however, no $\bar{\Lambda}$ anti-flow is measured, this would be a clear indication for a low $\bar{\Lambda}$ annihilation cross section and would thus support the above explanation for the $\bar{\Lambda}$ enhancement.

Alternatively the $\bar{\Lambda} / \overline{\mathrm{p}}$ enhancement could be explained by different medium modifications to the masses of non-strange and strange baryons. Introducing an additional attractive strange scalar condensate would lower the mass of $\bar{\Lambda}$ in hot and dense hadronic matter below that of $\bar{p}$ and thus account for $\bar{\Lambda} / \overline{\mathrm{p}}$ enhancement.

Sollfrank reviewed thoroughly the approaches of different groups to the understanding of particle yields. Most of them assume a thermodynamically equilibrated system and fit the temperature $T$ and chemical potential $\mu_{\mathrm{B}}$ of a free hadron gas to the particle ratios. From that they conclude which region in the phase diagram of hadronic matter spanned by $T$ and $\mu_{\mathrm{B}}$ is reached with current heavy-ion experiments and whether they are beyond the phase transition boundary or not. However, comparing the results of different groups, one finds a large uncertainty with $120<T<220$ and $150<\mu_{\mathrm{B}}<300$ for SPS energies. Additionally, up to now medium effects and hadronic interactions have not been taken into account within the calculations. Even if particle ratios can be fitted by purely hadronic models, it does not necessarily indicate that the system has always been in the hadronic phase. It might also have originated in the quark phase and then evolved along the phase boundary to the hadronic phase.

In contrast to Becattini, Sollfrank made dynamical calculations with hydrodynamics, and similarly he found that $\gamma_{\mathrm{s}}$ has to be taken to be less then one in order to describe $\mathrm{Pb}+\mathrm{Pb}$ data. This means that strangeness cannot saturate in a hydrodynamical approach where thermal equilibrium is assumed.

The Athens group including Kapoyiannis, Ktorides and Panagiotou extended the statistical bootstrap model in the strangeness dimension. With this they can predict particle ratios in the hadron gas and determine the critical surface in the space spanned by the temperature $T$ and quark chemical potentials $\mu_{q}$ and $\mu_{\mathrm{s}}$ where the hadronic world ceases to exist.

\subsection{Medium effects in strange matter}

Medium effects may play a significant role for the properties and formation of strange matter. Carsten Greiner reported on the influence of medium-dependent quark masses following 
from the hard dense loop approach on the stability of strange matter and the properties of strange stars. Strange quark matter is found to be less bound with a less strangeness content $f_{\mathrm{s}}$ compared to the common ansatz without inclusion of medium effects. In contrast, one does not find a considerable effect of medium modified quark masses, if the radiusmass relation of strange stars through solving the Oppenheimer-Volkoff-Tolman equations is analysed.

A different approach to the investigation of the hot and dense medium is pursued by Papazoglou: he constructs an effective $S U(3)_{L} \times S U(3)_{R}$ theory with broken scale invariance which incorporates spin- 0 , spin- 1 and spin- $\frac{1}{2}$ multiplets. The parameters are fixed to reproduce vacuum masses of hadrons and nuclear matter saturation properties. It turns out that finite nuclei are also described satisfactorily. Within this approach it is possible to calculate hadronic masses in the dense and hot medium as well as multiple strange nuclei. He finds that hyperons reduce their mass considerably at high densities/temperatures. Therefore, the minimum energy to create them is reduced, and an enhanced production of baryons and anti-baryons is possible.

\subsection{Quark-gluon and hadron dynamics}

Since it is not clear whether thermodynamical equilibrium has been reached in a heavyion reaction, a microscopic approach is necessary to account for the dynamics of nonequilibrated systems. Aichelin followed a such a dynamical approach: by solving transport equations on the Hartree level derived from the Nambu-Jona Lasinio model he studied the time evolution of a quark-anti-quark plasma. Starting from a quark phase, the quarkanti-quark pairs become rapidly more massive, i.e. they acquire a dynamical mass through interaction. This models the hadronization process starting from a quark phase which is possibly created in a heavy-ion collision. However, in that framework it is not clear how to explain the observed different freeze-out times for the various hadrons which all have a different mass.

The hadronization of quark matter has also been investigated by Zimanyi with the 'transchemistry model' which describes the transition of quark matter into hadron matter by calculating the production rate of hadrons from their constituent quarks assuming prescriptions for the elementary microscopic processes. By comparing multiplicities resulting from models assuming a different hadronization speed, with data from $\mathrm{SPS} \mathrm{Pb}+\mathrm{Pb}$ Zimanyi hopes to gain insight into the speed of hadronization.

Capella and Ferreiro discussed a mechanism of baryon stopping based on diquark breaking. They split the nucleon-nucleus cross section into its diquark preserving and diquark breaking components and found that the latter decreases faster with baryon number $A$ than the diquark preserving part of the cross section. Whereas there is little influence on the net $(\mathrm{p} \overline{\mathrm{p}})$ distribution for $\mathrm{NN}$ and peripheral SS collisions, the effect for central SS collisions is large. With this it is possible to describe the corresponding experimental data correctly, in contrast to the traditional approach where one takes an effective baryon fragmentation function determined from $\mathrm{pp}$ data and uses it in $\mathrm{p} A$ and $A B$ collisions.

\section{3. $\mathrm{J} / \Psi$ suppression}

A currently intensively discussed topic is the interpretation of the $\mathrm{J} / \Psi$ suppression observed by the NA50 collaboration in $\mathrm{Pb}+\mathrm{Pb}$ collisions. In the literature, there are two different interpretations of the observed $\mathrm{J} / \Psi$ suppression in heavy systems, and both can describe the $\mathrm{Pb}+\mathrm{Pb}$ data. In the conventional hadronic scenario the suppression results from the 


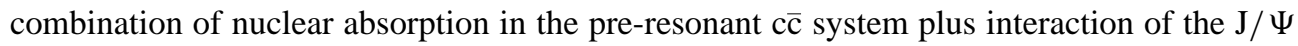
bound state with comovers. The second scenario is to assume the formation of a quarkgluon plasma. According to Vogt the $\mathrm{J} / \psi$ suppression observed in the $\mathrm{NA} 50 \mathrm{~Pb}+\mathrm{Pb}$ data can be explained if comovers are included in Glauber calculations. However, an observation of enhanced charm production beyond that predicted from initial nucleon-nucleon collisions would signal the onset of new physics which, according to Vogt, may be due to massive quark and gluon excitations.

\section{Astrophysics and strange matter}

A proposal for a measurable signal of the conversion of a 'neutron' star from the confined to the deconfined phase was given by Glendenning. Pulsars contain a huge amount of angular momentum and rotational energy. The emission of electromagnetic radiation and electronpositron pairs (over a time span of many years) causes a reduction in the angular velocity and thus also a reduction in the centrifugal deformation of the pulsar. Consequently, the interior density of the pulsar increases and may rise from below the critical density for a phase transition to above the critical value. In the resulting phase transition a conversion from rather incompressible nuclear matter to highly compressible quark matter will take place (starting at the centre of the pulsar), which reduces the radius of the pulsar and causes an anomalous decrease of the moment of inertia yielding an increase of the angular velocity. This is superposed on the normal reduction of angular velocity due to radiation loss. To conserve angular momentum, the deceleration of the angular velocity may decrease or even change sign resulting in a pulsar spin-up. The time span, in which this effect may be observable, is estimated to be of the order of $10^{5}$ years. Since the mean life of pulsars is around $10^{7}$ years, $1 \%$ of the 700 known pulsars may currently be undergoing this phase transition.

Schaab presented a thorough analysis of the cooling behaviour of neutron and strange stars, respectively. He found that the surface temperatures of strange stars is more or less indistinguishable from those of slowly cooling neutron stars. However, if a pulsar shortly after its formation in a supernova explosion were observed, which drops its temperature promptly, it would thus offer a signal of a strange star.

Christiansen showed that the presence of impurities during the cosmological quarkhadron transition leads to an dramatic increase of the mean nucleation distance $d_{\text {nuc }}$ to above $1 \mathrm{~m}$, in contrast to the usual expectation that impurities (these might be primordial black holes, magnetic monopoles, cosmic strings, etc) act as extra seeds for nucleation sites, therefore decreasing additionally the nucleation distance.

\section{Strangelet formation and stability}

Current experiments discussed at this meeting search for long-lived and slightly-charged strange matter droplets. Schaffner-Bielich argued that it is more likely to produce shortlived, highly negative charged strangelets $(Z / A=0.5)$ with a strangeness fraction greater than 1. The inclusion of shell effects reveals a strong binding of 'magic' strangelets which is due to a large spin-orbit splitting for quarks $(\approx 100 \mathrm{MeV})$. On the other side, metastable exotic multihypernuclear objects (MEMOs) might exist with a negative charge and positive baryon number, due to the replacement of protons with $\Xi^{-}$and $\Sigma^{-}$. In addition, the experiments planned at RHIC and LHC (see Coffin's talk) offer the possibility to detect multiple charmed objects, so called charmlets. Even with high bag constants, for which no strangelets would exist, bound charmed matter can be found. 


\section{Hypermatter experiments}

On which time scales do the strange exotic objects occur? All processes in the first $10^{-10} \mathrm{~s}$ are governed by strong interactions: after the initial reaction, the strangeness separation through the distillery mechanism takes place in the first $10^{-22} \mathrm{~s}$ after the collision. MEMOs are metastable and may exist therefore in the first $10^{-10} \mathrm{~s}$ before the weak decay $\Lambda \rightarrow \mathrm{N}+\pi$ sets in. Exotic strange objects which live beyond that time may be strange matter droplets, or strangelets. The experiments E864 and NA52 can only detect particles which live longer than $10^{-7} \mathrm{~s}$, i.e. long-lived strangelets.

Is it possible, however, to observe transient strange quark matter? To clarify this question one could perform Hanbury-Brown-Twiss interferometry of hyperons, for example $\Lambda$ and $\Xi$. The second possibility would be to do correlations of unlike particles. And, thirdly, one could study the decay in flight using the STAR, ALICE or CASTOR detectors.

First steps in this direction were presented by Ardouin. He studied $\mathrm{K}^{+} \mathrm{K}^{-}$correlations assuming that a quark matter bag with $A \approx 400$ was formed in a collision at AGS energies. Due to the distillery mechanism and the mutual Coulomb interaction of the unequal particles he could show that $\mathrm{K}^{-}$are emitted later than $\mathrm{K}^{+}$particles, on average.

Although the distillery mechanism is expected to work only in the baryon-rich region, Coffin argued that it might be possible to detect strangelets even with the STAR (at RHIC) and ALICE (at LHC) experiments. The reason for this is that fluctuations might lead to a sufficiently large chemical potential even if the net $\mu / T$ ratio is very low. The search will be for different kinds of exotic objects:

(i) stable or long-lived $\left(t>10^{-7} \mathrm{~s}\right)$ objects. They could be detected as a single track successively in the vertex detector and in the TPC;

(ii) metastable $\left(t<10^{-7} \mathrm{~s}\right)$ particles, which may be identified through their decay products;

(iii) unstable objects (identification possibly through correlation of the decay products).

\section{Conclusions}

The last year has seen enormous progress - the heavy-ion programs at Brookhaven and CERN have unambiguously established

- stopping [3,4];

- resonance matter [5];

- collective flow of baryons and mesons [6,7];

- (anti-)strange baryon enhancement [8-11];

- large HBT source radii [12-14];

- dilepton-enhancement below the $\rho$ mass $[15,16]$;

- $\mathrm{J} / \Psi$ suppression [17] in $\mathrm{Pb}+\mathrm{Pb}$ collisions.

This impressive list of achievements serves as indisputable and exciting proof that a truly novel form of highly-excited elementary matter has been created. The investigation of this new state of matter will be one of the key issues in high-energy nuclear physics for the coming years.

Further experimental efforts are underway. At the AGS, where temperatures extracted from particle spectra are $T>T_{\mathrm{C}}$ [18], the highest chances for the discovery of partonic degrees of freedom lie in the measurement of the flow excitation function and the massive efforts in the domain of the strangelet search. The investigation of the physics of high baryon density (i.e. partial restoration of chiral symmetry via mass shifts of vector mesons), for which the gold beam at the AGS seems ideally suited, is hindered, however, by the 
lack of experimental set-ups capable of measuring electromagnetic probes in $A A$ collisions. Since RHIC construction is under way, prospects for new worthwhile experiments in that sector are almost zero.

At the CERN/SPS interesting data come out of the domain of electromagnetic probes, strange particle yields (most importantly anti-hyperons) and heavy quarkonia. Energy densities estimated from rapidity distributions and temperatures extracted from particle spectra indicate that one is on the verge of entering the domain of deconfinement. The measured dielectron and dimuon excesses as well as the suppression of heavy quarkonia offer tantalizing evidence for new physics. The same holds true for the yields of antihyperons. With respect to HBT source radii the matter is not yet completely resolved (extensive and precise comparisons with hadronic and deconfinement model calculations have yet to be performed). Directed flow has also now been observed for hyperons-a flow excitation function, filling the gap between $10 \mathrm{GeV} /$ nucleon (AGS) and $160 \mathrm{GeV} /$ nucleon (SPS) would be immensely useful since flow is the observable most closely linked to the equation of state. Also the excitation function of particle yield ratios $(\pi / p, d / p, k / \pi \ldots)$ and, in particular, anti-hyperon yields, may be a sensitive probe of changes in the physics of the EOS. Most intriguing, however, seems the search for novel forms of $S U$ (3) matter, for example MEMOS, strangelets or even charmlets (SU(4)), which not only could signal the creation of true quark matter via a deconfinement phase transition, but are most interesting in themselves, enabling the extension of the periodic table of elements into hitherto unexplored dimensions. A strong experimental effort is needed in that direction.

\section{Acknowledgments}

I am grateful to P Papazoglou for creating this manuscript from my nearly unreadable notes, and to S Soff and S Bass for critically reading the manuscript.

\section{References}

[1] Spieles C et al 1997 Proc. Int. Conf.: Structure of Vacuum and Elementary Matter (10-16 March 1996, Wilderness/George, South Africa) ed H Stöcker, A Gallmann and J H Hamilton (Singapore: World Scientific) p 491

[2] Spieles C, Stöcker H and Greiner C e-print nucl-th/9704008, Z. Phys. C, submitted

[3] Videbaek F et al 1995 Nucl. Phys. A 590 249c

[4] Wienold T et al 1996 Nucl. Phys. A 61076

[5] Barrette J et al 1995 Phys. Lett. 351B 93

[6] Barrette J et al e-Print-Archive nucl-ex/9610006, Phys. Rev. C, submitted

[7] Peitzmann T et al 1997 QCD phase transitions Proc. Int. Workshop on Gross Properties of Nuclei and Nuclear Excitation XXV (Hirschegg, Kleinwalsertal, Austria, January 1997)

[8] Anderson E et al 1992 Phys. Lett. 294B 127

[9] Abatzis S et al 1994 Nucl. Phys. A 566 499c Abatzis S et al 1994 Nucl. Phys. A 566 491c Abatzis S et al 1994 Nucl. Phys. A 566 225c

[10] Alber T et al 1996 Phys. Lett. 366B 56

[11] Bormann C et al 1997 J. Phys. G: Nucl. Part. Phys. 231817

[12] Beker H et al 1994 Z. Phys. C 64209

[13] Sullivan J P et al 1993 Phys. Rev. Lett. 703000

[14] Miskowiec D et al 1996 Nucl. Phys. A 610227

[15] Agakichiev G et al 1995 Phys. Rev. Lett. 751272

[16] Ullrich T et al 1996 Nucl. Phys. A 610317

[17] Gonin M et al 1996 Nucl. Phys. A 610404

[18] Braun-Munzinger P, Stachel J, Wessels J P and Xu N 1996 Phys. Lett. 365B 1 